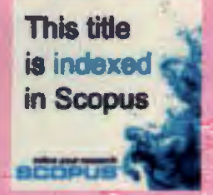

\title{
Reprinted from the
}

\section{Far East Journal of Mathematical Sciences (FJMS) Volume 99, Number 6, 2016, pp 813-830}

\section{THE HELICITY OF PEKERIS,}

ACCAD AND SHKOLLER FLOW

by

Tarhadi and Alhaji Akbar Bachtiar

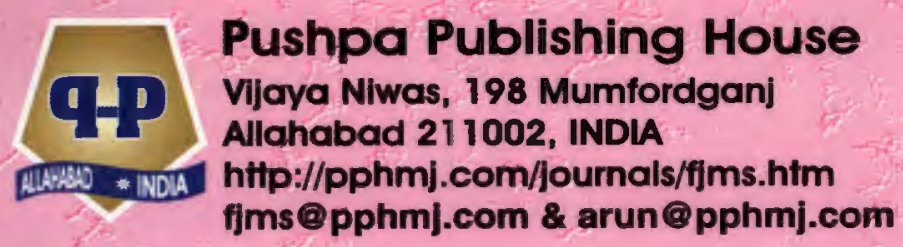




\section{Information for Authors}

Aims and Scope: The Far East Joumal of Mathematical Sciences (FJMS) is devoted to publishing original research papers and critical survey articles in the field of Pure and Applied Mathematics and Statistics. The FJMS is published in two volumes annually and each volume comprises of twelve issues. It is a fortnightly joumal.

Abstracting, Indexing and Reviews: Global Impact Factor : 0.987, Scopus, CrossRef DOIs databases, AMS Digital Mathematics Registry, ProQuest, IndexCopernicus, EBSCOhost, Zentralblatt MATH, Ulrich's web, Indian Science Abstracts, SCIRUS, OCLC, Excellence in Research for Australia (ERA), Google Scholar, AcademicKeys.

Submission of Manuscripts: Authors may submit their papers for consideration in the Far East Joumal of Mathematical Sciences (FJMS) by the following modes:

1. Online submission: Please visit journal's homepage at http://www.pphmj.com/journals/fjms.htm

2. Electronically: At the e-mail address: fjms@pphmj.com or kkazad@pphmj.com

3. Hard copies: Papers in duplicate with a letter of submission at the address of the publisher.

The paper must be typed only on one side in double spacing with a generous margin all round. An effort is made to publish a paper duly recommended by a referee within a period of three months. One set of galley proofs of a paper will be sent to the author submitting the paper, unless requested otherwise, without the original manuscript, for corrections.

Abstract and References: Authors are requested to provide an abstract of not more than 250 words and latest Mathematics Subject Classification. Statements of Lemmas, Propositions and Theorems should be set in italics and references should be arranged in alphabetical order by the surname of the first author.

Page Charges and Reprints: Authors are requested to arrange page charges of their papers @ USD 40.00 per page for USA and Canada, and EUR 30.00 per page for rest of the world from their institutions/research grants, if any. However, for authors in India this charge is Rs. 800.00 per page. No extra charges for printing colour figures. Twenty-five reprints in print version and a copy in soft version are provided to the corresponding author ex-gratis. Additional sets of reprints may be ordered at the time of proof correction.

Copyright: It is assumed that the submitted manuscript has not been published and will not be simultaneously submitted or published elsewhere. By submitting a manuscript, the authors agree that the copyright for their articles is transferred to the Pushpa Publishing House, Allahabad, India, if and when, the paper is accepted for publication. The publisher cannot take the responsibility of any loss of manuscript. Therefore, authors are requested to maintain a copy at their end.

\section{Subscription Information for 2016}

Institutional Price for all countries except India

\begin{tabular}{|l|r|r|}
\hline Electronic Subscription & $€ 905.00$ & US\$ 1195.00 \\
\hline Print Subscription includes Online Access & $€ 1295.00$ & US $\$ 1735.00$ \\
\hline
\end{tabular}

For Institutions: On seeking a license for volume(s) of the Far East Joumal of Mathematical Sciences (FJMS), the facility to download and print the articles will be available through the institutional 9 digits IP addreas to be provided by the appropriate authority. The facility to download will continue till the end of the next calendar year from the last issue of the volume subscribed. For having continued facility to keep the download of the sarne subscribed volume for another two calendar years mey be had on a considerable discounted rate.

Price in Indian Rs. (For Indian Institutions in India only)

\begin{tabular}{|l|l|}
\hline Print Subscription Only & Rs. 19500.00 \\
\hline
\end{tabular}

The subscription year runs from January 1, 2016 through December 31, 2016.

Information: The journals published by the "Pushpa Publishing House" are solely distributed by the "Vijaya Books and Journals Distributors".

Contact Person: Subscription Manager, Vijaya Books and Joumals Distributors, Vijaya Niwas, 198 Mumfordganj, Allahabad 211002, India; sub@pphmi.com; arun@pphmj.com 


\title{
THE HELICITY OF PEKERIS, ACCAD AND SHKOLLER FLOW
}

\author{
Tarhadi and Alhaji Akbar Bachtiar \\ Department of Mathematics Education \\ Universitas Terbuka \\ Indonesia \\ e-mail: tarhadi.fkip@gmail.com \\ Department of Mathematics \\ Universitas Indonesia \\ Indonesia \\ e-mail: alhaji1104@yahoo.com
}

\begin{abstract}
Helicity of PAS (Pekeris-Accad-Shkoller) flow is the scalar product of velocity $v$ flow and its curl, in a Euclidean space. Since PAS flow is a Beltrami flow with a property $C \nu=\operatorname{curl} \nu$, with $C$ is a constant, the helicity of PAS flow can be determined by integrating the scalar product between vectors $v$ and $v$ and multiply with $\Lambda$. This paper will explain the process of calculating the helicity of PAS.
\end{abstract}

Received: October 4, 2015; Revised: December: 9, 2015; Accepted: December 26, 2015 2010 Mathematics Subject Classification: 00A69.

Keywords and phrases: helicity, flow.

Communicated by Shahrdad G. Sajjadi; Editor: Advances and Applications in Fluid Mechanics: Published by Pushpa Publishing House. 


\section{Introduction}

PAS flow is defined by

$$
\begin{aligned}
& v=2 \mathcal{R}\left\{s_{2}^{2}+t_{2}^{2}\right\}, \\
& s_{2}^{2}=K \Lambda j_{2}(\Lambda r), \\
& t_{2}^{2}=\Lambda s_{2}^{2}(r), \\
& K=\sqrt{6 / 5},
\end{aligned}
$$

where $\Lambda$ is the positive root of the second order of Spherical Bessel function $j_{2}(r)$,

$$
j_{2}(r)=-\frac{3 \cos r}{r^{2}}+\frac{\left(3-r^{2}\right) \sin r}{r^{3}}
$$

and helicity of a flow is defined by [2],

$$
H=\int_{V} v \cdot \nabla \times v d V,
$$

while the property of Beltrami flow is [3],

$$
C v=\nabla \times v,
$$

So that the integral (1.5) can be substituted by the following form:

$$
H=C \int_{V} v \cdot v d V .
$$

It is easy to show that PAS flow has

$$
C=\Lambda
$$

so that the integral $(1.7)$ recast into

$$
H=\Lambda \int_{V} v \cdot v d V .
$$

\section{Main Results}

Based on (1.1), it can be stated that 


$$
v=s_{2}^{2}+s_{2}^{-2}+t_{2}^{2}+t_{2}^{-2}
$$

so that the integrand form in the integral (1.8) can be changed into

$$
\begin{aligned}
v \cdot v= & \left(s_{2}^{2}+s_{2}^{-2}+t_{2}^{2}+t_{2}^{-2}\right) \cdot\left(s_{2}^{2}+s_{2}^{-2}+t_{2}^{2}+t_{2}^{-2}\right) \\
= & s_{2}^{2} s_{2}^{2}+s_{2}^{2} s_{2}^{-2}+s_{2}^{2} t_{2}^{2}+s_{2}^{2} t_{2}^{-2}+s_{2}^{-2} s_{2}^{2}+s_{2}^{-2} s_{2}^{-2}+s_{2}^{-2} t_{2}^{2} \\
& +s_{2}^{-2} t_{2}^{-2}+t_{2}^{2} s_{2}^{2}+t_{2}^{2} s_{2}^{-2}+t_{2}^{2} t_{2}^{2}+t_{2}^{2} t_{2}^{-2}+t_{2}^{-2} s_{2}^{2}+t_{2}^{-2} s_{2}^{-2} \\
& +t_{2}^{-2} t_{2}^{2}+t_{2}^{-2} t_{2}^{-2}
\end{aligned}
$$

with

$$
d V=r^{2} \sin \theta d r d \theta d \phi
$$

and

$$
0 \leq r \leq 1,0 \leq \theta \leq \pi, 0 \leq \phi \leq 2 \pi .
$$

Integrands (2.1) are composed by 16 parts of scalar product between two vectors. To determine the integral solution of (1.8), the spherical harmonics function is needed to apply in it. This function is defined by [1],

$$
Y_{n}^{m}(\theta, \phi)=(-)^{m}\left(\frac{2 n+1}{2-\delta_{m}^{0}}\right)^{\frac{1}{2}} P_{n}^{m}(\cos \theta) e^{i m \phi}=(-)^{m} \overline{Y_{n}^{-m}},
$$

where $\overline{Y_{n}^{-m}}$ is conjugate of $Y_{n}^{m}(\theta, \phi)$,

$$
P_{n}^{m}(\cos \theta)=\left(\frac{2-\delta_{m}^{0}}{(n+m) !}\right)^{\frac{1}{2}} \frac{\left(1-\cos ^{2} \theta\right)}{2^{n} n !}\left[\frac{d}{d \cos \theta}\right]^{m+n}\left(\cos ^{2} \theta-1\right)^{n},
$$

is an associated Legendre polynomial with $n$ degree and $m$ order, with $0 \leq \theta$ $\leq \pi, e^{i m \phi}$ is Euler function with $0 \leq \phi \leq 2 \pi$,

$$
\delta_{m}^{0} \text { is delta Kronecker with } \delta_{m}^{0}=\left\{\begin{array}{l}
1, m=0 \\
0, m \neq 0
\end{array}\right.
$$


Because in PAS flow $m=2$ and $n=2$ then the integrand (2.1) are expanded into spherical harmonic functions of $Y_{2}^{2}$ and $Y_{2}^{-2}$, and since the integral of $\int_{0}^{2 \pi}\left(e^{i 2 \phi}\right)^{2} d \phi$ and $\int_{0}^{2 \pi}\left(e^{-i 2 \phi}\right)^{2} d \phi$ are zero, the $\phi$ integral, composed of $Y_{2}^{2} Y_{2}^{2}$, and $Y_{2}^{-2} Y_{2}^{-2}$ are zero. While the results of the $\phi$ integral which is composed by $Y_{2}^{2} Y_{2}^{-2}$, is $2 \pi$, because $\int_{0}^{2 \pi} e^{i 2 \phi} \cdot e^{-i 2 \phi} d \phi=\int_{0}^{2 \pi} d \phi=2 \pi$. By these properties of the $\phi$ integral, the 16 parts of integrand (2.1) can be reduced to

$$
s_{2}^{2} s_{2}^{-2}, s_{2}^{2} t_{2}^{-2}, s_{2}^{-2} s_{2}^{2}, s_{2}^{-2} t_{2}^{2}, t_{2}^{2} s_{2}^{-2}, t_{2}^{2} t_{2}^{-2}, t_{2}^{-2} s_{2}^{2}, t_{2}^{-2} t_{2}^{2}
$$

These reduced integrands are then be expanded in the form of spherical harmonic functions as the following.

First, we expand the pair integrand $s_{2}^{2} s_{2}^{-2}$ and $s_{2}^{-2} s_{2}^{2}$,

$$
\begin{aligned}
s_{2}^{2} s_{2}^{-2}= & \left(\frac{6}{r} s_{2}^{2} Y_{2}^{2}, \frac{1}{r} \frac{d}{d r}\left(r s_{2}^{2}\right) \frac{\partial Y_{2}^{2}}{\partial \theta}, \frac{1}{e \sin \theta} \frac{d}{d r}\left(r s_{2}^{2}\right) \frac{\partial Y_{2}^{2}}{\partial \phi}\right) \\
& \left(\frac{6}{r} s_{2}^{2} Y_{2}^{-2}, \frac{1}{r} \frac{d}{d r}\left(r s_{2}^{2}\right) \frac{\partial Y_{2}^{-2}}{\partial \theta}, \frac{1}{r \sin \theta} \frac{d}{d r}\left(r s_{2}^{2}\right) \frac{\partial Y_{2}^{-2}}{\partial \phi}\right) \\
= & \frac{36}{r^{2}}\left(s_{2}^{2}\right)^{2} Y_{2}^{2} Y_{2}^{-2}+\frac{1}{r^{2}}\left[\frac{d}{d r}\left(r s_{2}^{2}\right)\right]^{2} \frac{\partial Y_{2}^{2}}{\partial \theta} \frac{\partial Y_{2}^{-2}}{\partial \theta} \\
& +\frac{1}{r^{2} \sin ^{2} \theta}\left[\frac{d}{d r}\left(r s_{2}^{2}\right)\right]^{2} \frac{\partial Y_{2}^{2}}{\partial \phi} \frac{\partial Y_{2}^{-2}}{\partial \phi}, \\
s_{2}^{-2} s_{2}^{2}= & \left(\frac{6}{r} s_{2}^{2} Y_{2}^{-2}, \frac{1}{r} \frac{d}{d r}\left(r s_{2}^{2}\right) \frac{\partial Y_{2}^{-2}}{\partial \theta}, \frac{1}{r \sin \theta} \frac{d}{d r}\left(r s_{2}^{2}\right) \frac{\partial Y_{2}^{-2}}{\partial \phi}\right) \\
& \left(\frac{6}{r} s_{2}^{2} Y_{2}^{2}, \frac{1}{r} \frac{d}{d r}\left(r s_{2}^{2}\right) \frac{\partial Y_{2}^{2}}{\partial \theta}, \frac{1}{r \sin \theta} \frac{d}{d r}\left(r s_{2}^{2}\right) \frac{\partial Y_{2}^{2}}{\partial \phi}\right)
\end{aligned}
$$




$$
\begin{aligned}
= & \frac{36}{r^{2}}\left(s_{2}^{2}\right)^{2} Y_{2}^{-2} Y_{2}^{2}+\frac{1}{r^{2}}\left[\frac{d}{d r}\left(r s_{2}^{2}\right)\right]^{2} \frac{\partial Y_{2}^{-2}}{\partial \theta} \frac{\partial Y_{2}^{2}}{\partial \theta} \\
& +\frac{1}{r^{2} \sin ^{2} \theta}\left[\frac{d}{d r}\left(r s_{2}^{2}\right)\right]^{2} \frac{\partial Y_{2}^{-2}}{\partial \phi} \frac{\partial Y_{2}^{2}}{\partial \phi} .
\end{aligned}
$$

By the properties,

$$
\left.\begin{array}{l}
\frac{\partial Y_{2}^{2}}{\partial \theta} \frac{\partial Y_{2}^{-2}}{\partial \theta}=\frac{\partial Y_{2}^{-2}}{\partial \theta} \frac{\partial Y_{2}^{2}}{\partial \theta} \\
\frac{\partial Y_{2}^{2}}{\partial \phi} \frac{\partial Y_{2}^{-2}}{\partial \phi}=\frac{\partial Y_{2}^{-2}}{\partial \phi} \frac{\partial Y_{2}^{2}}{\partial \phi}
\end{array}\right\}
$$

then $s_{2}^{2} s_{2}^{-2}=s_{2}^{-2} s_{2}^{2}$

Second, we expand the pair integrand $t_{2}^{2} t_{2}^{-2}$ and $t_{2}^{-2} t_{2}^{2}$,

$$
\begin{aligned}
\boldsymbol{t}_{2}^{2} \boldsymbol{t}_{2}^{-2} & =\left(0, \frac{t_{2}^{2}}{\sin \theta} \frac{\partial Y_{2}^{2}}{\partial \phi},-t_{2}^{2} \frac{\partial Y_{2}^{2}}{\partial \theta}\right)\left(0, \frac{t_{2}^{2}}{\sin \theta} \frac{\partial Y_{2}^{-2}}{\partial \phi},-t_{2}^{2} \frac{\partial Y_{2}^{2}}{\partial \theta}\right) \\
& =\frac{\left(t_{2}^{2}\right)^{2}}{\sin ^{2} \theta} \frac{\partial Y_{2}^{2}}{\partial \phi} \frac{\partial Y_{2}^{-2}}{\partial \phi}+\left(-t_{2}^{2}\right)^{2} \frac{\partial Y_{2}^{2}}{\partial \theta} \frac{\partial Y_{2}^{-2}}{\partial \theta} \\
& =\frac{\left(t_{2}^{2}\right)^{2}}{\sin ^{2} \theta} \frac{\partial Y_{2}^{2}}{\partial \phi} \frac{\partial Y_{2}^{-2}}{\partial \phi}+\left(t_{2}^{2}\right)^{2} \frac{\partial Y_{2}^{2}}{\partial \theta} \frac{\partial Y_{2}^{-2}}{\partial \theta}, \\
\boldsymbol{t}_{2}^{-2} \boldsymbol{t}_{2}^{2} & =\left(0, \frac{t_{2}^{2}}{\sin \theta} \frac{\partial Y_{2}^{-2}}{\partial \phi},-t_{2}^{2} \frac{\partial Y_{2}^{-2}}{\partial \theta}\right)\left(0, \frac{t_{2}^{2}}{\sin \theta} \frac{\partial Y_{2}^{2}}{\partial \phi},-t_{2}^{2} \frac{\partial Y_{2}^{2}}{\partial \theta}\right) \\
& =\frac{\left(t_{2}^{2}\right)^{2}}{\sin ^{2} \theta} \frac{\partial Y_{2}^{-2}}{\partial \phi} \frac{\partial Y_{2}^{2}}{\partial \phi}+\left(-t_{2}^{2}\right)^{2} \frac{\partial Y_{2}^{-2}}{\partial \theta} \frac{\partial Y_{2}^{2}}{\partial \theta} \\
& =\frac{\left(t_{2}^{2}\right)^{2}}{\sin ^{2} \theta} \frac{\partial Y_{2}^{-2}}{\partial \phi} \frac{\partial Y_{2}^{2}}{\partial \phi}+\left(t_{2}^{2}\right)^{2} \frac{\partial Y_{2}^{-2}}{\partial \theta} \frac{\partial Y_{2}^{2}}{\partial \theta} .
\end{aligned}
$$

By the properties (2.6), equations (2.7) and (2.8) are equal so that $t_{2}^{2} t_{2}^{-2}=$ $t_{2}^{-2} t_{2}^{2}$ 
Third, we expand the pair integrands $s_{2}^{2} t_{2}^{-2}$ and $t_{2}^{2} s_{2}^{-2}$,

$$
\begin{aligned}
s_{2}^{2} t_{2}^{-2}= & \left(\frac{6}{r} s_{2}^{2} Y_{2}^{2}, \frac{1}{r} \frac{d}{d r}\left(r s_{2}^{2}\right) \frac{\partial Y_{2}^{2}}{\partial \theta}, \frac{1}{r \sin \theta} \frac{d}{d r}\left(r s_{2}^{2}\right) \frac{\partial Y_{2}^{2}}{\partial \phi}\right) \\
& \cdot\left(0, \frac{t_{2}^{2}}{\sin \theta} \frac{\partial Y_{2}^{-2}}{\partial \phi},-t_{2}^{2} \frac{\partial Y_{2}^{-2}}{\partial \theta}\right) \\
= & \frac{t_{2}^{2}}{r \sin \theta} \frac{d}{d r}\left(r s_{2}^{2}\right) \frac{\partial Y_{2}^{2}}{\partial \theta} \frac{\partial Y_{2}^{-2}}{\partial \phi}-\frac{t_{2}^{2}}{r \sin \theta} \frac{d}{d r}\left(r s_{2}^{2}\right) \frac{\partial Y_{2}^{2}}{\partial \phi} \frac{\partial Y_{2}^{-2}}{\partial \theta} \\
= & \frac{t_{2}^{2}}{r \sin \theta} \frac{d}{d r}\left(r s_{2}^{2}\right)\left(\frac{\partial Y_{2}^{2}}{\partial \theta} \frac{\partial Y_{2}^{-2}}{\partial \phi}-\frac{\partial Y_{2}^{2}}{\partial \phi} \frac{\partial Y_{2}^{-2}}{\partial \theta}\right), \\
\boldsymbol{t}_{2}^{2} s_{2}^{-2}= & \left(0, \frac{t_{2}^{2}}{\sin \theta} \frac{\partial Y_{2}^{2}}{\partial \phi},-t_{2}^{2} \frac{\partial Y_{2}^{2}}{\partial \theta}\right) \\
& \cdot\left(\frac{6}{r} s_{2}^{2} Y_{2}^{-2}, \frac{1}{r} \frac{d}{d r}\left(r s_{2}^{2}\right) \frac{\partial Y_{2}^{-2}}{\partial \theta}, \frac{1}{r \sin \theta} \frac{d}{d r}\left(r s_{2}^{2}\right) \frac{\partial Y_{2}^{-2}}{\partial \phi}\right) \\
= & \frac{t_{2}^{2}}{r \sin \theta} \frac{d}{d r}\left(r s_{2}^{2}\right) \frac{\partial Y_{2}^{2}}{\partial \phi} \frac{\partial Y_{2}^{-2}}{\partial \theta}-\frac{t_{2}^{2}}{r \sin \theta} \frac{d}{d r}\left(r s_{2}^{2}\right) \frac{\partial Y_{2}^{2}}{\partial \theta} \frac{\partial Y_{2}^{-2}}{\partial \phi}\left(\frac{\partial Y_{2}^{2}}{\partial \phi} \frac{\partial Y_{2}^{-2}}{\partial \theta}-\frac{\partial Y_{2}^{2}}{\partial \theta} \frac{\partial Y_{2}^{-2}}{\partial \phi}\right) .
\end{aligned}
$$

By the property,

$$
\frac{\partial Y_{2}^{2}}{\partial \theta} \frac{\partial Y_{2}^{-2}}{\partial \phi}=-\frac{\partial Y_{2}^{-2}}{\partial \theta} \frac{\partial Y_{2}^{2}}{\partial \phi}
$$

then equations (2.9) and (2.10) show that $s_{2}^{2} t_{2}^{-2}=-t_{2}^{2} s_{2}^{-2}$.

Fourth, we expand the pairs integrands $t_{2}^{-2} s_{2}^{2}$ and $t_{2}^{2} s_{2}^{-2}$,

$$
\boldsymbol{t}_{2}^{-2} \boldsymbol{s}_{2}^{2}=\left(0, \frac{t_{2}^{2}}{\sin \theta} \frac{\partial Y_{2}^{-2}}{\partial \phi},-t_{2}^{2} \frac{\partial Y_{2}^{-2}}{\partial \theta}\right)
$$




$$
\begin{aligned}
& \cdot\left(\frac{6}{r} s_{2}^{2} Y_{2}^{2}, \frac{1}{r} \frac{d}{d r}\left(r s_{2}^{2}\right) \frac{\partial Y_{2}^{2}}{\partial \theta}, \frac{1}{r \sin \theta} \frac{d}{d r}\left(r s_{2}^{2}\right) \frac{\partial Y_{2}^{2}}{\partial \phi}\right) \\
= & \frac{t_{2}^{2}}{r \sin \theta} \frac{d}{d r}\left(r s_{2}^{2}\right) \frac{\partial Y_{2}^{-2}}{\partial \phi} \frac{\partial Y_{2}^{-2}}{\partial \theta}-\frac{t_{2}^{2}}{r \sin \theta} \frac{d}{d r}\left(r s_{2}^{2}\right) \frac{\partial Y_{2}^{-2}}{\partial \theta} \frac{\partial Y_{2}^{2}}{\partial \phi} \\
= & \frac{t_{2}^{2}}{r \sin \theta} \frac{d}{d r}\left(r s_{2}^{2}\right)\left(\frac{\partial Y_{2}^{-2}}{\partial \phi} \frac{\partial Y_{2}^{2}}{\partial \theta}-\frac{\partial Y_{2}^{-2}}{\partial \theta} \frac{\partial Y_{2}^{2}}{\partial \phi}\right), \\
\boldsymbol{t}_{2}^{2} s_{2}^{-2}= & \left(0, \frac{t_{2}^{2}}{\sin \theta} \frac{\partial Y_{2}^{2}}{\partial \phi},-t_{2}^{2} \frac{\partial Y_{2}^{2}}{\partial \theta}\right) \\
& \cdot\left(\frac{6}{r} s_{2}^{2} Y_{2}^{-2}, \frac{1}{r} \frac{d}{d r}\left(r s_{2}^{2}\right) \frac{\partial Y_{2}^{-2}}{\partial \theta}, \frac{1}{r \sin \theta} \frac{d}{d r}\left(r s_{2}^{2}\right) \frac{\partial Y_{2}^{-2}}{\partial \phi}\right) \\
= & \frac{t_{2}^{2}}{r \sin \theta} \frac{d}{d r}\left(r s_{2}^{2}\right) \frac{\partial Y_{2}^{2}}{\partial \phi} \frac{\partial Y_{2}^{-2}}{\partial \theta}-\frac{t_{2}^{2}}{r \sin \theta} \frac{d}{d r}\left(r s_{2}^{2}\right) \frac{\partial Y_{2}^{2}}{\partial \theta} \frac{\partial Y_{2}^{-2}}{\partial \phi} \\
= & \frac{t_{2}^{2}}{r \sin \theta} \frac{d}{d r}\left(r s_{2}^{2}\right)\left(\frac{\partial Y_{2}^{2}}{\partial \phi} \frac{\partial Y_{2}^{-2}}{\partial \theta}-\frac{\partial Y_{2}^{2}}{\partial \theta} \frac{\partial Y_{2}^{-2}}{\partial \phi}\right) .
\end{aligned}
$$

According to the property (2.11), the results (2.12) and (2.13) show that $t_{2}^{-2} s_{2}^{2}=-t_{2}^{2} s_{2}^{-2}$.

From the expansions of integrands (2.1), therefore we find two pairs of integrands with same sign,

$$
s_{2}^{2} s_{2}^{-2}=s_{2}^{-2} s_{2}^{2},
$$

and

$$
\boldsymbol{t}_{2}^{2} \boldsymbol{t}_{2}^{-2}=\boldsymbol{t}_{2}^{-2} \boldsymbol{t}_{2}^{2},
$$

and two integrands with opposite sign,

$$
s_{2}^{2} t_{2}^{-2}=-t_{2}^{2} s_{2}^{-2}
$$

and

$$
t_{2}^{-2} s_{2}^{2}=-t_{2}^{2} s_{2}^{-2} \text {. }
$$


Since the sum of two integrands in (2.1) with opposite sign is zero, the sealar product of vector $v$ and $v$ in (2.1) can be reduced as the following:

$$
\begin{aligned}
v \cdot v & =s_{2}^{2} s_{2}^{-2}+s_{2}^{-2} s_{2}^{2}+t_{2}^{2} t_{2}^{-2}+t_{2}^{-2} t_{2}^{2} \\
& =2\left(s_{2}^{2} s_{2}^{-2}+t_{2}^{2} t_{2}^{-2}\right)
\end{aligned}
$$

Substitute (2.14) into (1.8), the integral form becomes

$$
\begin{aligned}
H & =\Lambda \int_{V} 2\left(s_{2}^{2} s_{2}^{-2}+t_{2}^{2} t_{2}^{-2}\right) d V \\
& =\Lambda \int_{0}^{2 \pi} \int_{0}^{\pi} \int_{0}^{1} 2\left(s_{2}^{2} s_{2}^{-2}+t_{2}^{2} t_{2}^{-2}\right) r^{2} \sin \theta d r d \theta d \phi .
\end{aligned}
$$

Substitute (2.4) and (2.7) into the integrand (2.15), will obtain

$$
\begin{aligned}
& 2\left(s_{2}^{2} s_{2}^{-2}+t_{2}^{2} t_{2}^{-2}\right) r^{2} \sin \theta \\
= & 2\left\{\left\{\frac{36}{r^{2}}\left(s_{2}^{2}\right)^{2} Y_{2}^{2} Y_{2}^{-2}\right.\right. \\
& \left.+\frac{1}{r^{2}}\left[\frac{d}{d r}\left(r s_{2}^{2}\right)\right]^{2} \frac{\partial Y_{2}^{2}}{\partial \theta} \frac{\partial Y_{2}^{-2}}{\partial \theta}+\frac{1}{r^{2} \sin ^{2} \theta}-\left[\frac{d}{d r}\left(r s_{2}^{2}\right)\right]^{2} \frac{\partial Y_{2}^{2}}{\partial \phi} \frac{\partial Y_{2}^{-2}}{\partial \phi}\right\} \\
& \left.+\left\{\frac{\left(t_{2}^{2}\right)^{2}}{\sin ^{2} \theta} \frac{\partial Y_{2}^{2}}{\partial \phi} \frac{\partial Y_{2}^{-2}}{\partial \phi}+\left(t_{2}^{2}\right)^{2} \frac{\partial Y_{2}^{2}}{\partial \theta} \frac{\partial Y_{2}^{-2}}{\partial \theta}\right\}\right) r^{2} \sin \theta \\
= & 2\left\{\frac{36}{r^{2}}\left(s_{2}^{2}\right)^{2} Y_{2}^{2} Y_{2}^{-2}\right. \\
& \left.+\frac{1}{r^{2}}\left[\frac{d}{d r}\left(r s_{2}^{2}\right)\right]^{2} \frac{\partial Y_{2}^{2}}{\partial \theta} \frac{\partial Y_{2}^{-2}}{\partial \theta}+\frac{1}{r^{2} \sin ^{2} \theta}\left[\frac{d}{d r}\left(r s_{2}^{2}\right)\right]^{2} \frac{\partial Y_{2}^{2}}{\partial \phi} \frac{\partial Y_{2}^{-2}}{\partial \phi}\right\} r^{2} \sin \theta \\
& +2\left\{\frac{\left(t_{2}^{2}\right)^{2}}{\sin ^{2} \theta} \frac{\partial Y_{2}^{2}}{\partial \phi} \frac{\partial Y_{2}^{-2}}{\partial \phi}+\left(t_{2}^{2}\right)^{2} \frac{\partial Y_{2}^{2}}{\partial \theta} \frac{\partial Y_{2}^{-2}}{\partial \theta}\right\} r^{2} \sin \theta
\end{aligned}
$$




$$
\begin{aligned}
= & \underbrace{72\left(s_{2}^{2}\right)^{2} Y_{2}^{2} Y_{2}^{-2} \sin \theta}_{1}+\underbrace{2 \sin \theta\left[\frac{d}{d r}\left(r s_{2}^{2}\right)\right]^{2} \frac{\partial Y_{2}^{2}}{\partial \theta} \frac{\partial Y_{2}^{-2}}{\partial \theta}}_{2} \\
& +\underbrace{\frac{2}{\sin \theta\left[\frac{d}{d r}\left(r s_{2}^{2}\right)\right]^{2} \frac{\partial Y_{2}^{2}}{\partial \phi} \frac{\partial Y_{2}^{-2}}{\partial \phi}}}_{3} \\
& +\underbrace{\frac{2 r^{2}\left(t_{2}^{2}\right)^{2}}{\sin \theta} \frac{\partial Y_{2}^{2}}{\partial \phi} \frac{\partial Y_{2}^{-2}}{\partial \phi}}_{4}+\underbrace{2 r^{2}\left(t_{2}^{2}\right)^{2} \sin \theta \frac{\partial Y_{2}^{2}}{\partial \theta} \frac{\partial Y_{2}^{-2}}{\partial \theta}}_{5} .
\end{aligned}
$$

So the integral (2.14) is the sum of five integrals with the integrand 1 until 5 in (2.16). To solve the integral (2.15), we expand these integrands to the following spherical harmonic functions form.

Integrand 1

$$
72\left(s_{2}^{2}\right)^{2} Y_{2}^{2} Y_{2}^{-2} \sin \theta=135 K^{2} \Lambda^{2}\left(j_{2}(\Lambda r)\right)^{2} \sin ^{4} \theta \sin \theta .
$$

Integrand 2

$$
\begin{aligned}
& 2 \sin \theta\left[\frac{d}{d r}\left(r s_{2}^{2}\right)\right]^{2} \frac{\partial Y_{2}^{2}}{\partial \theta} \frac{\partial Y_{2}^{-2}}{\partial \theta} \\
= & 2\left(K^{2} \Lambda^{4} r^{2}\left(j_{1}(\Lambda r)\right)^{2}-4 K^{2} \Lambda^{3} r j_{1}(\Lambda r) j_{2}(\Lambda r)\right. \\
& \left.+4 K^{2} \Lambda^{2}\left(j_{2}(\Lambda r)\right)^{2}\right)\left(\frac{15}{2} \sin ^{2} \theta \cos ^{2} \theta \sin \theta\right)
\end{aligned}
$$

Integrand 3

$$
\begin{aligned}
& \frac{2}{\sin \theta}\left[\frac{d}{d r}\left(r s_{2}^{2}\right)\right]^{2} \frac{\partial Y_{2}^{2}}{\partial \phi} \frac{\partial Y_{2}^{-2}}{\partial \phi} \\
= & 2\left(K^{2} \Lambda^{4} r^{2}\left(j_{1}(\Lambda r)\right)^{2}-4 K^{2} \Lambda^{3} r j_{1}(\Lambda r) j_{2}(\Lambda r)\right. \\
& \left.+4 K^{2} \Lambda^{2}\left(j_{2}(\Lambda r)\right)^{2}\right)\left(30 \cos ^{2} \theta \sin \theta\right) .
\end{aligned}
$$


Integrand 4

$$
\frac{2 r^{2}\left(t_{2}^{2}\right)^{2}}{\sin \theta} \frac{\partial Y_{2}^{2}}{\partial \phi} \frac{\partial Y_{2}^{-2}}{\partial \phi}=2 r^{2} K^{2} \Lambda^{4}\left(j_{2}(\Lambda r)\right)^{2}\left(30 \cos ^{2} \theta \sin \theta\right)
$$

Integrand 5

$$
2 r^{2}\left(t_{2}^{2}\right)^{2} \sin \theta \frac{\partial Y_{2}^{2}}{\partial \theta} \frac{\partial Y_{2}^{-2}}{\partial \theta}=2 r^{2} K^{2} \Lambda^{4}\left(j_{2}(\Lambda r)\right)^{2} \sin \theta\left(\frac{15}{2}\right) \sin ^{2} \theta \cos ^{2} \theta
$$

The next step is, to integrate each form of integrands from (2.17) until (2.21).

Since the three variables of integral, $\phi, \theta, r$ are mutually independent in the integral (2.15), we solve the integral as the multiplication of $\left(I_{\phi}\right)\left(I_{\theta}\right)\left(I_{r}\right)$. These integrals are associated to every part of the five integrands (2.16). We solve the integrals as the following order.

Integral $1, I_{1}$ with integrand (2.17).

Integral $\phi$,

$$
I_{1 \phi}=\int_{0}^{2 \pi} d \phi=2 \pi
$$

Integral $\theta$,

$$
I_{1 \theta}=\int_{0}^{\pi} \sin ^{4} \theta \sin \theta d \theta=\frac{16}{15}
$$

Integral $r$,

$$
I_{1 r}=\int_{0}^{1} 135 K^{2} \Lambda^{2}\left(j_{2}(\Lambda r)\right)^{2} d r
$$

So the result is integral 1 is

$$
I_{1}=\left(I_{1 \phi}\right)\left(I_{1 \theta}\right)\left(I_{1 r}\right)=288 \pi \int_{0}^{1} K^{2} \Lambda^{2}\left(j_{2}(\Lambda r)\right)^{2} d r
$$

Integral 2, $I_{2}$ with integrand (2.18). 
Integral $\phi$,

$$
I_{2 \phi}=\int_{0}^{2 \pi} d \phi=2 \pi
$$

Integral $\theta$,

$$
I_{2 \theta}=\int_{0}^{\pi} \frac{15}{2} \sin ^{2} \theta \cos ^{2} \theta \sin \theta d \theta=2 .
$$

Integral $r$,

$$
\begin{aligned}
I_{2 r}= & \int_{0}^{1} 2\left(K^{2} \Lambda^{4} r^{2}\left(j_{1}(\Lambda r)\right)^{2}-4 K^{2} \Lambda^{3} r j_{1}(\Lambda r) j_{2}(\Lambda r)\right. \\
& \left.+4 K^{2} \Lambda^{2}\left(j_{2}(\Lambda r)\right)^{2}\right) d r .
\end{aligned}
$$

Result integral 2 is

$$
\begin{aligned}
I_{2}=\left(I_{2 \phi}\right)\left(I_{2 \theta}\right)\left(I_{2 r}\right)= & 8 \pi \int_{0}^{1}\left(K^{2} \Lambda^{4} r^{2}\left(j_{1}(\Lambda r)\right)^{2}\right. \\
& \left.-4 K^{2} \Lambda^{3} r j_{1}(\Lambda r) j_{2}(\Lambda r)+4 K^{2} \Lambda^{2}\left(j_{2}(\Lambda r)\right)^{2}\right) d r .
\end{aligned}
$$

Integral $3, I_{3}$ with integrand (2.19).

Integral $\phi$,

$$
I_{3 \phi}=\int_{0}^{2 \pi} d \phi=2 \pi
$$

Integral $\theta$,

$$
I_{3 \theta}=\int_{0}^{\pi} \sin ^{3} \theta d \theta=\frac{4}{3} .
$$

Integral $r$,

$$
\begin{aligned}
I_{3 r}= & \int_{0}^{1} 15\left(K^{2} \Lambda^{4} r^{2}\left(j_{1}(\Lambda r)\right)^{2}-4 K^{2} \Lambda^{3} r j_{1}(\Lambda r) j_{2}(\Lambda r)\right. \\
& \left.+4 K^{2} \Lambda^{2}\left(j_{2}(\Lambda r)\right)^{2}\right) d r .
\end{aligned}
$$


Result integral 3 is

$$
\begin{aligned}
I_{3}=\left(I_{3 \phi}\right)\left(I_{3 \theta}\right)\left(I_{3 r}\right)= & 40 \pi \int_{0}^{1}\left(K^{2} \Lambda^{4} r^{2}\left(j_{1}(\Lambda r)\right)^{2}\right. \\
& \left.-4 K^{2} \Lambda^{3} r j_{1}(\Lambda r) j_{2}(\Lambda r)+4 K^{2} \Lambda^{2}\left(j_{2}(\Lambda r)\right)^{2}\right) d r .
\end{aligned}
$$

Integral $4, I_{4}$ with integrand (2.20).

Integral $\phi$,

$$
I_{4 \phi}=\int_{0}^{2 \pi} d \phi=2 \pi
$$

Integral $\theta$,

$$
I_{4 \theta}=\int_{0}^{\pi} \sin ^{3} \theta d \theta=\frac{4}{3} .
$$

Integral $r$,

$$
I_{4 r}=\int_{0}^{1} 15 K^{2} \Lambda^{4} r^{2}\left(j_{2}(\Lambda r)\right)^{2} d r
$$

Result integral 4 is

$$
I_{4}=\left(I_{4 \phi}\right)\left(I_{4 \theta}\right)\left(I_{4 r}\right)=40 \pi \int_{0}^{1} K^{2} \Lambda^{4} r^{2}\left(j_{2}(\Lambda r)\right)^{2} d r .
$$

Integral $5, I_{5}$ with integrand (2.21).

Integral $\phi$,

$$
I_{5 \phi}=\int_{0}^{2 \pi} d \phi=2 \pi
$$

Integral $\theta$,

$$
I_{5 \theta}=\int_{0}^{\pi} \frac{15}{2} \sin ^{2} \theta \cos ^{2} \theta \sin \theta d \theta=2 .
$$


Integral $r$,

$$
I_{5 r}=\int_{0}^{1} 2 K^{2} \Lambda^{4} r^{2}\left(j_{2}(\Lambda r)\right)^{2} d r .
$$

Result integral 5 is

$$
I_{5}=\left(I_{5 \phi}\right)\left(I_{5 \theta}\right)\left(I_{5 r}\right)=8 \pi \int_{0}^{1} K^{2} \Lambda^{4} r^{2}\left(j_{2}(\Lambda r)\right)^{2} d r .
$$

With these five results we can formulate the helicity of PAS flow as

$$
H=\Lambda\left(I_{1}+I_{2}+I_{3}+I_{4}+I_{5}\right)=\Lambda I_{1}+\Lambda I_{2}+\Lambda I_{3}+\Lambda I_{4}+\Lambda I_{5} .
$$

Hence,

$$
\begin{aligned}
H= & \Lambda\left(288 \pi \int_{0}^{1} K^{2} \Lambda^{2}\left(j_{2}(\Lambda r)\right)^{2} d r\right) \\
+ & \Lambda\left(8 \pi \int _ { 0 } ^ { 1 } \left(K^{2} \Lambda^{4} r^{2}\left(j_{1}(\Lambda r)\right)^{2}-4 K^{2} \Lambda^{3} r j_{1}(\Lambda r) j_{2}(\Lambda r)\right.\right. \\
& \left.\left.+4 K^{2} \Lambda^{2}\left(j_{2}(\Lambda r)\right)^{2}\right) d r\right) \\
& +\Lambda\left(4 0 \pi \int _ { 0 } ^ { 1 } \left(K^{2} \Lambda^{4} r^{2}\left(j_{1}(\Lambda r)\right)^{2}-4 K^{2} \Lambda^{3} r j_{1}(\Lambda r) j_{2}(\Lambda r)\right.\right. \\
& \left.\left.+4 K^{2} \Lambda^{2}\left(j_{2}(\Lambda r)\right)^{2}\right) d r\right) \\
& +\Lambda\left(40 \pi \int_{0}^{1} K^{2} \Lambda^{4} r^{2}\left(j_{2}(\Lambda r)\right)^{2} d r\right)+\Lambda\left(8 \pi \int_{0}^{1} K^{2} \Lambda^{4} r^{2}\left(j_{2}(\Lambda r)\right)^{2} d r\right) \\
= & \Lambda\left(288 \pi \int_{0}^{1} K^{2} \Lambda^{4}\left(j_{2}(\Lambda r)\right)^{2} d r\right) \\
& +\Lambda\left(4 8 \pi \int _ { 0 } ^ { 1 } \left(K^{2} \Lambda^{4} r^{2}\left(j_{1}(\Lambda r)\right)^{2}-4 K^{2} \Lambda^{3} r j_{1}(\Lambda r) j_{2}(\Lambda r)\right.\right. \\
& \left.\left.+4 K^{2} \Lambda^{2}\left(j_{2}(\Lambda r)\right)^{2}\right) d r\right)+\Lambda\left(48 \pi \int_{0}^{1} K^{2} \Lambda^{4} r^{2}\left(j_{2}(\Lambda r)\right)^{2} d r\right)
\end{aligned}
$$




$$
\begin{aligned}
= & 288 \pi \int_{0}^{1} K^{2} \Lambda^{3}\left(j_{2}(\Lambda r)\right)^{2} d r+48 \pi \int_{0}^{1} K^{2} \Lambda^{5} r^{2}\left(j_{1}(\Lambda r)\right)^{2} d r \\
& -192 \pi \int_{0}^{1} K^{2} \Lambda^{4} r j_{1}(\Lambda r) j_{2}(\Lambda r) d r \\
& +192 \pi \int_{0}^{1} K^{2} \Lambda^{3}\left(j_{2}(\Lambda r)\right)^{2} d r+48 \pi \int_{0}^{1} K^{2} \Lambda^{5} r^{2}\left(j_{2}(\Lambda r)\right)^{2} d r \\
= & 480 \pi \int_{0}^{1} K^{2} \Lambda^{3}\left(j_{2}(\Lambda r)\right)^{2} d r+48 \pi \int_{0}^{1} K^{2} \Lambda^{5} r^{2}\left(j_{1}(\Lambda r)\right)^{2} d r \\
& +48 \pi \int_{0}^{1} K^{2} \Lambda^{5} r^{2}\left(j_{2}(\Lambda r)\right)^{2} d r-192 \pi \int_{0}^{1} K^{2} \Lambda^{4} r j_{1}(\Lambda r) j_{2}(\Lambda r) d r .
\end{aligned}
$$

Write the integrals of (2.22) as

$$
\begin{aligned}
H= & \int_{0}^{1}\left(480 \pi K^{2} \Lambda^{3}\left(j_{2}(\Lambda r)\right)^{2}+48 \pi K^{2} \Lambda^{5} r^{2}\left(j_{1}(\Lambda r)\right)^{2}\right. \\
& \left.+48 \pi K^{2} \Lambda^{5} r^{2}\left(j_{2}(\Lambda r)\right)^{2}-192 \pi K^{2} \Lambda^{4} r j_{1}(\Lambda r) j_{2}(\Lambda r)\right) d r
\end{aligned}
$$

The integral of (2.23) is a globally helicity, which is calculated in a volume of a spherical space. The helicity could also calculated as a function of radius of a ball with $r$ between 0 and 1 .

This function is the integrand of integral (2.23), which is formulated as

$$
\begin{aligned}
H(r)= & 480 \pi K^{2} \Lambda^{3}\left(j_{2}(\Lambda r)\right)^{2}+48 \pi K^{2} \Lambda^{5} r^{2}\left(j_{1}(\Lambda r)\right)^{2} \\
& +48 \pi K^{2} \Lambda^{5} r^{2}\left(j_{2}(\Lambda r)\right)^{2}-192 \pi K^{2} \Lambda^{4} r j_{1}(\Lambda r) j_{2}(\Lambda r), \quad 0<r \leq 1 .
\end{aligned}
$$

We solve numerically the integral by software of Wolfram Mathematica 7, version 7.0.1. Since $K=\sqrt{6 / 5}, K^{2}=1.2$, so the numerical calculation of (2.23) is written in code 
NIntegrate $\left[480 * N[\mathrm{Pi}] * 1.2 * \Lambda^{\wedge} 3\right.$

* SphericalBesselJ $[2, \Lambda * \mathrm{r}]^{\wedge} 2+48 * N[\mathrm{Pi}] * 1.2 * \Lambda^{\wedge} 5 * \mathrm{r}^{\wedge} 2$

${ }^{*}$ SphericalBesselJ $[1, \Lambda * \mathrm{r}]^{\wedge} 2+48 * N[\mathrm{Pi}] * 1.2 * \Lambda^{\wedge} 5 * \mathrm{r}^{\wedge} 2$

* SphericalBesselJ $[2, \Lambda * \mathrm{r}]^{\wedge} 2-192 * N[\mathrm{Pi}] * 1.2 * \Lambda^{\wedge} 4 * \mathrm{r}$

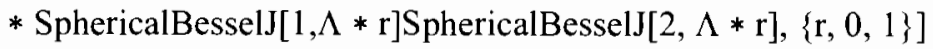

and the graph of (2.24) is written with code

$$
\begin{aligned}
\text { Plot } & {\left[480 N[\mathrm{Pi}] * 1.2 * \Lambda^{\wedge} 3\right.} \\
& * \text { SphericalBesselJ }[2, \Lambda * r]^{\wedge} 2+48 * N[\mathrm{Pi}] * 1.2 * \Lambda^{\wedge} 5 * r^{\wedge} 2 \\
& * \text { SphericalBesselJ }[1, \Lambda * r]^{\wedge} 2+48 * N[\mathrm{Pi}] * 1.2 * \Lambda^{\wedge} 5 * r^{\wedge} 2 \\
& * \text { SphericalBesselJ }[2, \Lambda * r]^{\wedge} 2-192 * N[\mathrm{Pi}] * 1.2 * \Lambda^{\wedge} 4 * r \\
& * \text { SphericalBesselJ }[1, \Lambda * r] \text { SphericalBesselJ }[2, \Lambda * r], \\
& \{r, 0,1\}, \text { Frame } \rightarrow \text { True, GridLines } \rightarrow * \text { Automatic }]
\end{aligned}
$$

Since the model of PAS flow, use $\Lambda=12.3229$ [5], the outcomes of integral (2.23) would be generated by the following codes:

$$
\text { NIntegrate }\left[480 * N[\mathrm{Pi}] * 1.2 * 12.3229^{\wedge} 3\right.
$$

* SphericalBesselJ $[2,12.3229 * r]^{\wedge} 2+48 * N[\mathrm{Pi}] * 1.2 * 12.3229 \wedge 5 * r^{\wedge} 2$

$*$ SphericalBesselJ $[1,12.3229 * r]^{\wedge} 2+48 * N[\mathrm{Pi}] * 1.2 * 12.3229^{\wedge} 5 * r^{\wedge} 2$

* SphericalBesselJ $[2,12.3229 * r]^{\wedge} 2-192 * N[\mathrm{Pi}] * 1.2 * 12.3229^{\wedge} 4 * r$

* SphericalBesselJ[1,12.3229*r]SphericalBesselJ[2,12.3229*r],

$$
\{r, 0,1\}]
$$

and the outcomes is $H=331932$.

The graph (2.24) is generated by the following code

$$
\text { Plot }\left[480 * N[\mathrm{Pi}] * 1.2 * 12.3229^{\wedge} 3\right.
$$

* SphericalBesselJ $[2,12.3229 * r]^{\wedge} 2+48 * N[\mathrm{Pi}] * 1.2 * 12.3229^{\wedge} 5 * r^{\wedge} 2$ 
* SphericalBesselJ $[1,12.3229 * r]^{\wedge} 2+48 * N[\mathrm{Pi}] * 1.2 * 12.3229^{\wedge} 5 * r^{\wedge} 2$

* SphericalBesselJ $[2,12.3229 * r]^{\wedge} 2-192 * N[\mathrm{Pi}] * 1.2 * 12.3229^{\wedge} 4 * r$

* SphericalBesselJ[1,12.3229*r]SphericalBesselJ[2,12.3229*r],

$\{r, 0,1\}$, Frame $\rightarrow$ True, GridLines $\rightarrow$ Automatic $]$

and the result is the following graph:

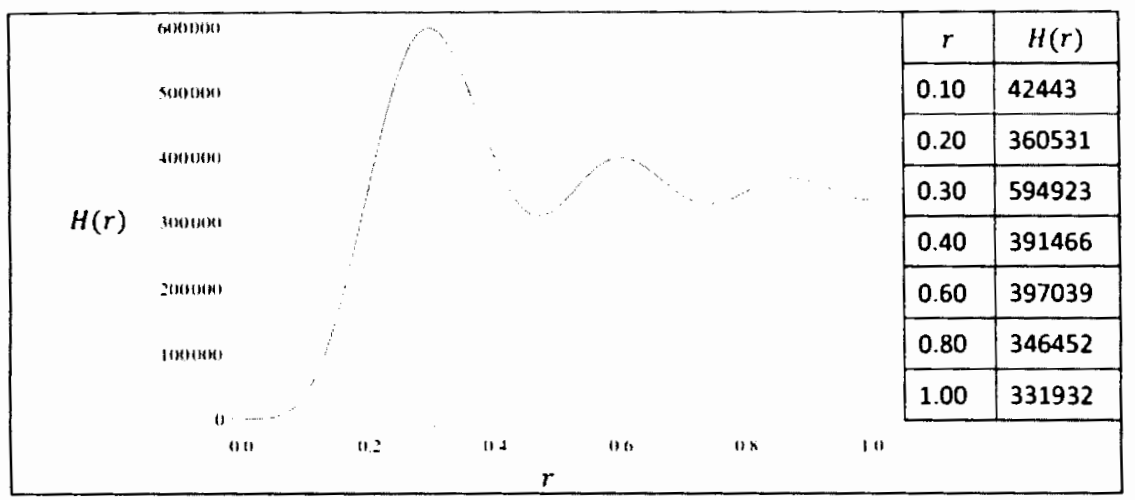

Graph of Helicity of PAS Flow with $\Lambda=12.3229$.

The table in the right side of the graph shows the value of $H(r)$ in a certain value of $r$, which is calculated as

$$
N\left[480 * N[\mathrm{Pi}] * 1.2 * 12.3229^{\wedge} 3\right.
$$

* SphericalBesselJ $[2,12.3229 * r]^{\wedge} 2+48 * N[\mathrm{Pi}] * 1.2^{*} 12.3229^{\wedge} 5 * r^{\wedge} 2$

* SphericalBesselJ $[1,12.3229 * r]^{\wedge} 2+48 * N[\mathrm{Pi}] * 1.2 * 12.3229^{\wedge} 5 * r^{\wedge} 2$

* SphericalBesselJ[2,12.3229*r $]^{\wedge} 2-192 * N[\mathrm{Pi}] * 1.2 * 12.3229^{\wedge} 4 * \mathrm{r}$

* SphericalBesselJ[1,12.3229*r]SphericalBesselJ[2,12.3229*r]]

As examples, we substitute $r$ with the values of $0.10,0.20,0.40,0.60,0.80$, and 1.00. Then the values of $H(r)$ are $H(0.10)=42443, H(0.20)=360531$, $H(0.40)=391446, H(0,60)=397039, H(0.80)=346452$, and $H(1.00)=$ 331932. 


\section{Conclusion}

The helicity of any flow is determined by integral that defined by Moffatt,

$$
H=\int_{V} v \cdot \nabla \times v d V
$$

Since PAS flow is Beltrami flow, which $v$ vector is parallel to its vorticity, $C v=\nabla \times v$, we can substitute the above integral to the following integral:

$$
H=\int_{V} v \cdot C v d V
$$

where $C$ is a constant, so that the integral becomes

$$
H=C \int_{V} v \cdot v d V
$$

If we determine the PAS flow directly by the definition of integral in (1.5), we have to expand vector $v$ and $\nabla \times v$, and then expand the scalar product of them. However, if we use the Beltrami property to determine the helicity of PAS flow, we need only to expand the product scalar between vector $v$ and the same vector. Therefore, Beltrami property make simpler in determining the helicity of PAS flow. With Beltrami property, we can derive that the value of $C$ which is equal to $\Lambda$, the positive roots of second order of Bessel function.

The result of global helicity calculated in a space of spherical volume and calculated as a function of radius of a ball are equal when $r=1$, that is $H=$ $H(1)=331932$.

\section{Acknowledgement}

The authors thank the anonymous referees for their valuable suggestions which let to the improvement of the manuscript. 


\section{References}

[1] A. A. Bachtiar, A study of planar velocity dynamos and related issues, Ph.D. Thesis, The University of Sidney, Sydney, 2009.

[2] H. K. Moffatt, Helicity in laminar and turbulent flow, Ann. Rev. Mech. 24 (1992), 281-312.

[3] H. K. Moffatt, Magnetic Field Generation in Electricity Conducting Fluids, Cambridge University Press. 1978.

[4] A. Jeffrey, Handbook of Mathematical Formulas and Integrals, 2nd ed., Academic Press, 2000.

[5] C. L. Pekeris, Y. Accad and B. Shkoller, Kinematics dynamos and the Earth's magnetic field, Proc. R. Soc. Lond. A 275 (1973), 425-461. 


\section{FAR EAST JOURNAL OF MATHEMATICAL SCIENCES (FJMS)}

\section{Editorial Board}

Editor-in-Chief: K. K. Azad, India

\section{Associate Editors:}

George S. Androulakis, Greece

Carlo Bardaro, Italy

Manoj Chanagt, India

Claudio Cuevas, Brazil

Maslina Darus, Malaysia

Massimiliano Ferrara, Italy

Salvatore Ganci, Italy

Demetris P. K. Ghikas, Greece

Lisa M. James, USA

Young Bae Jun, South Korea

Hideo Kojima, Japan

Alison Marr, USA

Haruhide Matsuda, Japan

Jon Seo Park, South Korea

Alexandre J. Santana, Brazil

Pooja Singh, India

A. L. Smirnov, Russian Federation

Chun-Lei Tang, China

Carl A. Toews, USA

Vladimir Tulovsky, USA

G. Brock Williams, USA

Chaohui Zhang, USA

Kewen Zhao, China
Natig M. Atakishiyev, Mexico

Antonio Carbone, Italy

Yong Gao Chen, China

Zhenlu Cui, USA

Manav Das, USA

Shusheng Fu, China

Wei Dong Gao, China

Jay M. Jahangiri, USA

Moonja Jeong, South Korea

Koji Kikuchi, Japan

Victor N. Krivtsov, Russian Federation

Dania Masood, India

Manouchehr Misaghian, USA

Cheon Seoung Ryoo, South Korea

K. P. Shum, China

Varanasi Sitaramaiah, India

Ashish K. Srivastava, USA

E. Thandapani, India

B. C. Tripathy, India

Qing-Wen Wang, China

Xiao-Jun Yang, China

Pu Zhang, China 


\section{OUR PUBLICATIONS}

1. Advances and Applications in Discrete Mathematics (GIF: 0.654)

2. Advances ard Applications in Fluid Mechanics (GIF : 1.345 ; SCÓpUS)

3. Advances and Applications in Statistics (GIF : 1.045)

4. Advances in Computer Science and Engineering (GIF : 1,023)

5. Advances in Differential Equations and Control Processes (GIF : 0.861 )

6. Advances in Fuzzy Sets and Systems (GIF : 1.009)

7. Current Development in Oceanography (GIF : 0.762)

8. Far East Journal of Applied Mathematics (GIF : 1.008)

9. Far East Journal of Dywamical Systems (GIF : 1.009)

10. Far East Journal of Electrentics and Communications (GIF:0.987; SCOPUS)

11. Far East Journal of Mathematical Education (GIF : 0.987)

12. Far East Journal of Mathematical Sciences (FMS) (GIF : 0.987 ; SCOPUS)

13. Far East Journal of Theoretical Statistics (GIF; 0.987)

14. International Journal of Eunctional Analysis, Operator Theory and Applications (GIF : 0.745)

15. International Journal of Materials Engineering and Technology (GIF : 1.234)

16. International Journal of Numerical Methods and Applications (GIF : 0.798)

17. International Journal of Nutrition and Dietetics

18. IP Journal of Algebra; Number Theory \& Applications (GIF : 0.786 ; SCOPUS)

19. JP Journal of Biostatistics (GIF : 0.897)

20. JP Journal of Fixed Point theory and Applications (GIF : 0.786)

21. JP Journal of Geometry and Topology (GIF : 0.987 ; SCOPUS)

22. JP Jourial of Heat and Mass Transfer (GIF : 0.876 ; SCOPUS)

23. Universal Journal of Mathematies and Mathematical Scienees. (GIF :0.745)

Pushpa Publishing House, Vijaya Niwas, 198 Mumfordgauj, Allahabad 211002, India arun@pphmj.com foww. pphmj.com 